\title{
Closed formula for the relative entropy of entanglement
}

\author{
Adam Miranowicz ${ }^{1}$ and Satoshi Ishizaka ${ }^{2,3}$ \\ ${ }^{1}$ Faculty of Physics, Adam Mickiewicz University, 61-614 Poznań, Poland \\ ${ }^{2}$ Nano Electronics Research Laboratories, NEC Corporation, 34 Miyukigaoka, Tsukuba 305-8501, Japan \\ ${ }^{3}$ INQIE, the University of Tokyo, 4-6-1 Komaba, Meguro-ku, Tokyo 153-8505, Japan
}

(Dated: October 31, 2018)

\begin{abstract}
The long-standing problem of finding a closed formula for the relative entropy of entanglement (REE) for two qubits is addressed. A compact-form solution to the inverse problem, which characterizes an entangled state for a given closest separable state, is obtained. Analysis of the formula for a large class of entangled states strongly suggests that a compact analytical solution of the original problem, which corresponds to finding the closest separable state for a given entangled state, can be given only in some special cases. A few applications of the compact-form formula are given to show additivity of the REE, to relate the REE with the Rains upper bound for distillable entanglement, and to show that a Bell state does not have a unique closest separable state.
\end{abstract}

PACS numbers: 03.67.Mn, 03.65.Ud, 42.50.Dv

\section{INTRODUCTION}

The relative entropy of entanglement (REE) is as an entanglement measure quantifying how much a given entangled state can be distinguished operationally from the set of separable states or those with positive partial transposition (PPT) [1]:

$$
E_{R}(\rho)=\min _{\sigma^{\prime} \in \mathcal{D}} S\left(\rho \| \sigma^{\prime}\right)=S(\rho \| \sigma),
$$

where $\mathcal{D}$ denotes a set of separable states or PPT states, $S$ is a quasidistance measure usually chosen to be the quantum relative entropy, $S\left(\rho \| \sigma^{\prime}\right)=\operatorname{tr}\left(\rho \lg \rho-\rho \lg \sigma^{\prime}\right)$, an analog of the classical Kullback-Leibler divergence. A state $\sigma$ on the boundary of separable states is called the closest separable state (CSS) or the closest PPT state. Various properties of the REE have already been described (see, e.g., [1, 2, 3, 4, 45, 6, 77, 8, 9, 10, 11, 12, 13, 14, 15, 16] and for a review see 17]). But there is still an open fundamental problem [18]: the task of finding a closed explicit formula for the REE for a given two-qubit state corresponding to a solution of the convex optimization problem for the REE, or, briefly, of finding the CSS $\sigma$ for a given entangled state $\rho$.

Here, we give a compact-form solution of the closely related problem of finding entangled states $\rho$ and their REE for a given CSS $\sigma$. Our formula is derived from the results obtained by one of us in Ref. [13]. We also demonstrate the intrinsic difficulty in solving the original problem. In addition, we apply our formula to relate the REE with the Rains upper bound for the distillable entanglement, and to show additivity of the REE and nonuniqueness of the CSS for a Bell state.

\section{A CLOSED FORMULA FOR THE REE}

Let us consider an entangled two-qubit state $\rho$ with its $\operatorname{CSS} \sigma$, and assume that $\sigma$ is full rank. Note that the case includes the arbitrary full-rank entangled state $\rho$, since $\operatorname{rank}(\sigma) \geq \operatorname{rank}(\rho)$ must hold so that $S(\rho \| \sigma)$ is finite, and hence the CSS $\sigma$ for full-rank $\rho$ is always full rank. Since $\sigma$ is an edge state then its partial transposition $\sigma^{\Gamma}$ is rank deficient, i.e., $\operatorname{rank}\left(\sigma^{\Gamma}\right)=3$. Let $|\phi\rangle$ be the kernel (or null space) of $\sigma^{\Gamma}$, i.e., an eigenstate of $\sigma^{\Gamma}$ corresponding to zero eigenvalue,

$$
\sigma^{\Gamma}|\phi\rangle=0
$$

Moreover, let $|i\rangle$ and $\lambda_{i}$ be eigenstates and eigenvalues of $\sigma$, respectively. Using the kernel $|\phi\rangle$, the formula in Ref. [13] can be rewritten in the following simple form:

$$
\rho=\sigma-x G(\sigma)
$$

where

$$
\begin{array}{r}
G(\sigma)=\sum_{i j} G_{i j}|i\rangle\left\langle i\left|(|\phi\rangle\langle\phi|)^{\Gamma}\right| j\right\rangle\langle j|, \\
G_{i j} \equiv\left\{\begin{array}{cc}
\lambda_{i} & \text { for } \lambda_{i}=\lambda_{j}, \\
\frac{\lambda_{i}-\lambda_{j}}{\lg \lambda_{i}-\lg \lambda_{j}} & \text { for } \lambda_{i} \neq \lambda_{j},
\end{array}\right.
\end{array}
$$

and $x \geq 0$. All $\rho$ obtained from Eq. (3) for $x_{\max } \geq x>0$ have $\sigma$ as their CSS, where $x_{\max }$ is the threshold for $\rho \geq 0$. This is a unique solution of all extremal conditions in two qubits as shown later. The same relation holds for entangled states and the closest PPT state even in higher-dimensional systems as long as the (PPT) entanglement witness (EW) $Z$ such that $\operatorname{tr} Z \sigma=0$ is uniquely determined as $(|\phi\rangle\langle\phi|)^{\Gamma}$. Moreover, from Eqs. (3) -(5) we have

$$
\langle i|\rho| i\rangle=\lambda_{i}\left[1-x\left\langle i\left|(|\phi\rangle\langle\phi|)^{\Gamma}\right| i\right\rangle\right] .
$$

Therefore, the REE can be rewritten as

$$
\begin{aligned}
E_{R}(\rho)= & \operatorname{tr} \rho \lg \rho-\operatorname{tr} \rho \lg \sigma \\
= & \operatorname{tr} \rho \lg \rho-\sum_{i}\langle i|\rho| i\rangle \lg \lambda_{i} \\
= & \operatorname{tr} \rho \lg \rho-\operatorname{tr} \sigma \lg \sigma \\
& +x \sum_{i}\left\langle i\left|(|\phi\rangle\langle\phi|)^{\Gamma}\right| i\right\rangle \lambda_{i} \lg \lambda_{i} \\
= & S(\sigma)-S(\rho)+x \operatorname{tr}\left[(|\phi\rangle\langle\phi|)^{\Gamma} \sigma \lg \sigma\right],
\end{aligned}
$$


where $S(\cdot)$ is the von Neumann entropy. In any case, however, Eq. (3) should be conversely solved with respect to $\sigma$ to obtain the true closed formula for the REE as a solution to Eisert's problem [18]. In the following we show that the inversion is possible in some special cases but rather not in general.

Our formula can be derived from the results obtained in Ref. [13] but the derivation is rather lengthy. Fortunately, we can also derive it in a compact and elegant way using a different approach based on the result shown in Ref. [19] that the operator of

$$
Z=I-\int_{0}^{\infty} \frac{1}{\sigma+z} \rho \frac{1}{\sigma+z} d z
$$

must be an entanglement witness. Let us briefly repeat the proof in Ref. [19] for our later convenience. Since $\sigma$ is the CSS for $\rho$, the inequality

$$
S\left(\rho \|(1-\epsilon) \sigma+\epsilon \sigma^{\prime}\right)-S(\rho \| \sigma) \geq 0
$$

must hold for every separable state $\sigma^{\prime}$ and $0 \leq \epsilon \leq 1$. Using the expansion of

$$
\lg (X+\epsilon Y)=\lg (X)+\epsilon \int_{0}^{\infty} \frac{1}{X+z} Y \frac{1}{X+z} d z+\mathcal{O}\left(\epsilon^{2}\right)
$$

we have

$$
\begin{aligned}
& S\left(\rho \|(1-\epsilon) \sigma+\epsilon \sigma^{\prime}\right)-S(\rho \| \sigma) \\
& \quad=\epsilon \operatorname{tr} \rho \int_{0}^{\infty} \frac{1}{\sigma+z}\left(\sigma-\sigma^{\prime}\right) \frac{1}{\sigma+z} d z+\mathcal{O}\left(\epsilon^{2}\right) \\
& \quad=\epsilon\left[\operatorname{tr} \rho-\operatorname{tr} \sigma^{\prime} \int_{0}^{\infty} \frac{1}{\sigma+z} \rho \frac{1}{\sigma+z} d z\right]+\mathcal{O}\left(\epsilon^{2}\right) \\
& \quad=\epsilon \operatorname{tr} Z \sigma^{\prime}+\mathcal{O}\left(\epsilon^{2}\right),
\end{aligned}
$$

and hence $\epsilon \operatorname{tr} Z \sigma^{\prime}+\mathcal{O}\left(\epsilon^{2}\right) \geq 0$ must hold for arbitrary small $\epsilon>0$ if $\sigma^{\prime}$ is a separable state. This implies that $\operatorname{tr} Z \sigma^{\prime} \geq 0$ must hold for every separable state $\sigma^{\prime}$, and therefore $Z$ must be an EW [19].

Now suppose that $\sigma$ and $\rho$ are two-qubit states. It was shown in Ref. [20] that an EW in two qubits must be decomposable, since there are no PPT entangled states in two qubits. Therefore, $Z$ must be a decomposable EW, and hence $Z=P+Q^{\Gamma}$, where $P$ and $Q$ are positive operators [20]. Moreover,

$\operatorname{tr} Z \sigma=\operatorname{tr}\left[\sigma-\rho \int_{0}^{\infty} \frac{1}{\sigma+z} \sigma \frac{1}{\sigma+z} d z\right]=\operatorname{tr}(\sigma-\rho)=0$,

and as a result $\operatorname{tr} Z \sigma=\operatorname{tr} P \sigma+\operatorname{tr} Q \sigma^{\Gamma}=0$ must hold. Since $\sigma$ is full rank, and $\sigma^{\Gamma}$ is positive and rank 3 , the solution is uniquely determined (leaving out the normalization of $x>0)$ as $P=0$ and $Q=x|\phi\rangle\langle\phi|$. As a result, $x(|\phi\rangle\langle\phi|)^{\Gamma}=Z$ holds for full rank $\sigma$. The integral in $Z$ can be performed using the eigenstates $|i\rangle$ for $\sigma$ such that

$$
\begin{aligned}
x\left\langle i\left|(|\phi\rangle\langle\phi|)^{\Gamma}\right| j\right\rangle & =\delta_{i j}-\int_{0}^{\infty} \frac{1}{\lambda_{i}+z}\langle i|\rho| j\rangle \frac{1}{\lambda_{j}+z} d z \\
& =\delta_{i j}-\langle i|\rho| j\rangle G_{i j}^{-1}
\end{aligned}
$$

and finally we have Eq. (3). In this way, the satisfaction of all extremal conditions in the optimization problem for the REE is automatically ensured by the condition that $Z$ is an EW such that $\operatorname{tr} Z \sigma=0$. Note that $|\phi\rangle$ is always entangled for full rank $\sigma$ [21] (if $|\phi\rangle$ is not entangled, the full rank $\sigma$ is not a CSS for any entangled state).

\section{THE RAINS BOUND AND THE REE}

An upper bound for distillable entanglement introduced by Rains [6] is defined as

$$
R(\rho)=\min _{\tau^{\prime} \geq 0}\left[S\left(\rho \| \tau^{\prime}\right)+\lg \operatorname{tr}\left|\tau^{\prime \Gamma}\right|\right],
$$

where minimization is taken over all states including entangled states, and hence $R(\rho) \leq E_{R}(\rho)$ follows from the definition. Here, let us apply the technique as used in the previous section to the optimization problem for the Rains bound. It was shown in Ref. [11] that the problem is reduced to

$$
R(\rho)=\min _{\tau^{\prime} \geq 0} S\left(\rho \| \tau^{\prime}\right)
$$

where the minimization is taken over unnormalized states subject to $\operatorname{tr}\left|\tau^{\prime \Gamma}\right| \leq 1$. This is a convex optimization problem because $\operatorname{tr}\left|\tau_{0}^{\Gamma}\right| \leq 1$ for $\tau_{0}=p \tau_{1}+(1-p) \tau_{2}$ and $\operatorname{tr}\left|\tau_{1}^{\Gamma}\right|, \operatorname{tr}\left|\tau_{2}^{\Gamma}\right| \leq 1$ [11]. Suppose that $\rho$ is full rank and $\tau$ is an optimal unnormalized state. Hence, $\tau$ is full rank, $\operatorname{tr}\left|\tau^{\Gamma}\right|=1$, and

$$
S\left(\rho \|(1-\epsilon) \tau+\epsilon \tau^{\prime}\right)-S(\rho \| \tau) \geq 0
$$

for every $\tau^{\prime}$ such that $\operatorname{tr}\left|\tau^{\prime \Gamma}\right| \leq 1$ and $0 \leq \epsilon \leq 1$. Let $\tau^{\prime}$ be a normalized separable state, i.e., $\tau^{\prime \Gamma} \geq 0$ and $\operatorname{tr}\left|\tau^{\prime \Gamma}\right|=$ $\operatorname{tr} \tau^{\prime}=1$. Using the expansion of the logarithmic function for $\epsilon \rightarrow+0$ as in the previous section, we have

$$
\begin{aligned}
& S\left(\rho \|(1-\epsilon) \tau+\epsilon \tau^{\prime}\right)-S(\rho \| \tau) \\
& \quad \sim \epsilon \operatorname{tr}\left[I-\int_{0}^{\infty} \frac{1}{\tau+z} \rho \frac{1}{\tau+z} d z\right] \tau^{\prime} \\
& \quad \equiv \epsilon \operatorname{tr} Z_{R} \tau^{\prime} \geq 0,
\end{aligned}
$$

and hence $Z_{R}$ must be again an EW. Note however that $\operatorname{tr} Z_{R} \tau=\operatorname{tr} \tau-\operatorname{tr} \rho \leq 0$ in this case, contrary to Eq. (11), because $\tau$ is unnormalized so that $\operatorname{tr}\left|\tau^{\Gamma}\right|=1$.

Let us then consider the case where $\rho$ is a two-qubit state, and suppose that the optimal two-qubit state $\tau$ is entangled. Since the partial transposition of a two-qubit state has only one negative eigenvalue [9] $\tau^{\Gamma}$ is expressed such that

$$
\tau^{\Gamma}=(1-\mu) \Pi-\mu|\psi\rangle\langle\psi|,
$$

where $\Pi \geq 0, \Pi|\psi\rangle=0$, and $\mu>0$. Moreover, $\operatorname{tr} \Pi=1$ so that $\operatorname{tr}\left|\tau^{\Gamma}\right|=1$. For a small deviation of $\mu \rightarrow(1+\delta) \mu$, we have

$$
\begin{aligned}
S(\rho \| \tau) & \rightarrow S(\rho \| \tau)-\delta \mu\left[\operatorname{tr} Z_{R}\left(\Pi^{\Gamma}+(|\psi\rangle\langle\psi|)^{\Gamma}\right)-2\right] \\
& =S(\rho \| \tau)-\delta\left[\operatorname{tr} Z_{R}\left(\Pi^{\Gamma}-\tau\right)-2 \mu\right]
\end{aligned}
$$




$$
=S(\rho \| \tau)-\delta \operatorname{tr} Z_{R} \Pi^{\Gamma},
$$

where $\operatorname{tr} Z_{R} \tau=\operatorname{tr} \tau-1=-2 \mu$ was used. Since $\tau$ must satisfy the extremal condition with respect to $\mu$, $\operatorname{tr} Z_{R} \Pi^{\Gamma}=0$ must hold. Moreover, since $\tau$ is a twoqubit entangled state, $\Pi$ is rank-3 and $\Pi^{\Gamma}$ is positive definite [14], and as a result the EW $Z_{R}$ is uniquely determined (including the normalization in this case) as $Z_{R}=2(|\psi\rangle\langle\psi|)^{\Gamma}$, where $\operatorname{tr} Z_{R} \tau=-2 \mu$ and $\mu \neq 0$ were taken into account. This implies that

$$
I-2(|\psi\rangle\langle\psi|)^{\Gamma}=\int_{0}^{\infty} \frac{1}{\tau+z} \rho \frac{1}{\tau+z} d z,
$$

but this cannot be satisfied because the right-hand side is positive definite for full-rank $\rho$ and $\tau$, while the lefthand side is not for any $|\psi\rangle$. Therefore, the optimal state $\tau$ must not be entangled, and the optimization in $R(\rho)$ is achieved by a separable state. The same discussion also holds for low-rank $\rho$, because $R(\rho)$ is a continuous function [11]. It is then concluded that $R(\rho)=E_{R}(\rho)$ for every two-qubit state, and our compact-form formula also holds for the Rains bound.

Note that the Rains bound is strictly smaller than the REE for the Werner state in higher-dimensional systems [5, [6], but such a disagreement does not occur in two qubits as shown above.

\section{ADDITIVITY OF THE REE}

An asymptotic REE defined as

$$
E_{R}^{\infty}(\rho)=\lim _{n \rightarrow \infty} \frac{1}{n} E_{R}\left(\rho^{\otimes n}\right)
$$

satisfies $E_{R}^{\infty}(\rho) \leq E_{R}(\rho)$ from the definition. The equality holds if $E_{R}(\rho)$ is weakly additive, but this is not the case in general [5]. Here, let us briefly investigate the additivity using our compact-form formula.

In Ref. [26], it was shown that $E_{R}(\rho)$ for $\rho$ such that $[\rho, \sigma]=0$ is weakly additive if $\left(\rho \sigma^{-1}\right)^{\Gamma} \geq-\mathbb{1}$. From Eq. (3),

$$
\begin{aligned}
& \left\langle i\left|\left(\lg \lambda_{i} \rho-\rho \lg \lambda_{j}\right)\right| j\right\rangle \\
& \quad=-x\left\langle i\left|\left[\lambda_{i}(|\phi\rangle\langle\phi|)^{\Gamma}-(|\phi\rangle\langle\phi|)^{\Gamma} \lambda_{j}\right]\right| j\right\rangle
\end{aligned}
$$

must hold for all $i$ and $j$, and hence we have $[\lg \sigma, \rho]=$ $-x\left[\sigma,(|\phi\rangle\langle\phi|)^{\Gamma}\right]$. This implies that $[\rho, \sigma]=0$ if and only if $\left[\sigma,(|\phi\rangle\langle\phi|)^{\Gamma}\right]=0$. Therefore, $(|\phi\rangle\langle\phi|)^{\Gamma}$ must be diagonalized in terms of the eigenstates of $\sigma$ so that $[\rho, \sigma]=0$, and the compact-form formula in this case is much simplified as

$$
\rho=\sigma-x(|\phi\rangle\langle\phi|)^{\Gamma} \sigma .
$$

Let $p_{0} \geq 1 / 2$ be the maximal Schmidt coefficient of $|\phi\rangle$. Since the largest eigenvalue of $(|\phi\rangle\langle\phi|)^{\Gamma}$ is $p_{0}$, the range of $x$ must satisfy $0 \leq x \leq 1 / p_{0} \leq 2$, so that $\rho \geq 0$. As a result, $\left(\rho \sigma^{-1}\right)^{\Gamma}=I-x|\phi\rangle\langle\phi| \geq-\mathbb{1}$ always holds. Therefore, it is concluded that $E_{R}(\rho)$ is weakly additive and $E_{R}^{\infty}(\rho)=E_{R}(\rho)$ for every two-qubit state such that $[\rho, \sigma]=0$.

Moreover, it was shown in Ref. [26] that $E_{R}(\rho)$ for $\rho$ such that $[\rho, \sigma]=0$ is strongly additive, namely $E_{R}(\rho \otimes$ $\tau)=E_{R}(\rho)+E_{R}(\tau)$ for an arbitrary $\tau$, if $\left(\rho \sigma^{-1}\right)^{\Gamma} \geq 0$. In the same way as above, it is found that the condition is satisfied if $x \leq 1$, and therefore $E_{R}(\rho)$ is strongly additive for every two-qubit state such that $[\rho, \sigma]=0$ and $x \leq 1$.

\section{FORMULA APPLICATIONS FOR FULL-RANK CSS}

All the examples of arbitrary rank states $\rho$ with their CSS $\sigma$ found by us in the literature [1, 2, 8, 9, 10, 11, 15, 16], can easily be explained using our formula. The procedure can be summarized as follows: choose a full-rank matrix $\sigma$, calculate its partial transposition to get $\sigma^{\Gamma}$, find a condition on its elements for which $\sigma^{\Gamma}$ is rank deficient (and so becomes a CSS), calculate $\rho=\sigma-x G(\sigma)$, if required take a limit of some elements to diminish the rank of $\sigma$ and $\rho$, and finally find an inverse relation to express the elements of $\sigma$ in terms of those of $\rho$.

For example, let us analyze a full-rank state $\sigma=$ $\sum_{i=1}^{4} R_{i}\left|\beta_{i}\right\rangle\left\langle\beta_{i}\right|$ diagonal in the Bell basis $\left\{\left|\beta_{i}\right\rangle\right\}$. The eigenvalues of $\sigma^{\Gamma}$ are $\Lambda_{i}=\frac{1}{2}-R_{i}$. Thus, e.g., by setting $\Lambda_{1}=0, \sigma$ becomes a CSS. By noting that the kernel $|\phi\rangle$ is a Bell state and applying it to Eq. (3), one gets a Bell-diagonal entangled state $\rho_{\mathrm{BD}}=\sum_{i} r_{i}\left|\beta_{i}\right\rangle\left\langle\beta_{i}\right|$, where $r_{1}=(2+x) / 4$ and otherwise $r_{i}=R_{i}(1-x / 2)$. By inverting the latter equation, one gets the well-known formula [1]

$$
\sigma_{\mathrm{BD}}=\frac{1}{2}\left|\beta_{1}\right\rangle\left\langle\beta_{1}\left|+\frac{1}{2\left(1-r_{1}\right)} \sum_{i=2}^{4} r_{i}\right| \beta_{i}\right\rangle\left\langle\beta_{i}\right| .
$$

This is the CSS of an arbitrary Bell-diagonal state $\rho_{\mathrm{BD}}=$ $\sum_{i=1}^{4} r_{i}\left|\beta_{i}\right\rangle\left\langle\beta_{i}\right|$ assuming $r_{1} \geq 1 / 2$.

As another example which, to our knowledge, has not been discussed in the literature, let us analyze a twoqubit state of the following form:

$$
\sigma_{\mathrm{Z}}=\left(\begin{array}{cccc}
R_{1} & 0 & 0 & 0 \\
0 & R_{2} & Y & 0 \\
0 & Y & R_{3} & 0 \\
0 & 0 & 0 & R_{4}
\end{array}\right)
$$

This state is the CSS if its partial transposition $\sigma_{\mathrm{Z}}^{\Gamma}$ is rank 3 , which implies that $Y=\sqrt{R_{1} R_{4}} e^{i \varphi}$ (in the following we set $\varphi=0$ ), while the requirement of positivity of the density operator $\sigma_{\mathrm{Z}}$ implies that $R_{2} R_{3} \geq Y^{2}$. Thus, $\rho_{\mathrm{Z}}$ satisfies the condition $R_{2} R_{3} \geq R_{1} R_{4}$, and (24) can compactly be given by

$$
\sigma_{\mathrm{Z}}=\mathcal{N}(|\psi\rangle\langle\psi|)^{\Gamma}+R_{2}|01\rangle\left\langle 01\left|+R_{3}\right| 10\right\rangle\langle 10|,
$$

where $|\psi\rangle=\mathcal{N}^{-1 / 2}\left(\sqrt{R_{1}}|00\rangle+\sqrt{R_{4}}|11\rangle\right)$ and $\mathcal{N}=R_{1}+$ $R_{4}$. The eigenvalues of $\sigma_{\mathrm{Z}}$ are $\left(\lambda_{i}\right)_{i}=\left(R_{1}, R_{4}, \lambda_{+}, \lambda_{-}\right)$, where $\lambda_{ \pm}=\frac{1}{2}\left(R_{2}+R_{3} \pm z\right)$ with the auxiliary function $z=\sqrt{\left(R_{2}-R_{3}\right)^{2}+4 Y^{2}}$. The corresponding eigenvectors are $\left|\lambda_{1}\right\rangle=|00\rangle,\left|\lambda_{2}\right\rangle=|11\rangle$, and $\left|\lambda_{ \pm}\right\rangle=$ 
$\mathcal{N}_{ \pm}\left[\left(\lambda_{ \pm}-R_{3}\right)|01\rangle+Y|10\rangle\right]$ with normalizations $\mathcal{N}_{ \pm}=$ $\left[\left(\lambda_{ \pm}-R_{3}\right)^{2}+Y^{2}\right]^{-1 / 2}$. One finds the kernel $|\phi\rangle$ of $\sigma_{\mathrm{Z}}^{\Gamma}$ and then

$$
\begin{array}{r}
(|\phi\rangle\langle\phi|)^{\Gamma}=\left(R_{1}+R_{4}\right)^{-1}\left(R_{4}|00\rangle\langle 00|-Y| 01\rangle\langle 10|\right. \\
\left.-Y|10\rangle\left\langle 01\left|+R_{1}\right| 11\right\rangle\langle 11|\right) .
\end{array}
$$

Thus, according to (4), we find that

$$
G\left(\sigma_{\mathrm{Z}}\right)=\left(\begin{array}{cccc}
\bar{R}_{1} & 0 & 0 & 0 \\
0 & \bar{R}_{2} & \bar{Y} & 0 \\
0 & \bar{Y} & \bar{R}_{3} & 0 \\
0 & 0 & 0 & \bar{R}_{4}
\end{array}\right)
$$

where

$$
\begin{aligned}
\bar{R}_{1} & =\bar{R}_{4}=\frac{Y^{2}}{R_{1}+R_{4}}, \\
\bar{R}_{2} & =-2 Y^{2} d\left[\left(R_{2}-R_{3}\right)\left(z+R_{2} L\right)+2 Y^{2} L\right], \\
\bar{R}_{3} & =-2 \bar{R}_{1}-\bar{R}_{2}, \\
\bar{Y} & =Y d\left[2 Y^{2}\left(R_{2}+R_{3}\right) L-\left(R_{2}-R_{3}\right)^{2} z\right]
\end{aligned}
$$

are given in terms of

$$
\begin{aligned}
z & =\sqrt{\left(R_{2}-R_{3}\right)^{2}+4 R_{1} R_{4}}, \\
L & =\ln \left(R_{2}+R_{3}-z\right)-\ln \left(R_{2}+R_{3}+z\right),
\end{aligned}
$$

and $1 / d=\left(R_{1}+R_{4}\right) z^{2} L$.

Thus, according to (3), the entangled states

$$
\rho_{\mathrm{Z}}=\sigma_{\mathrm{Z}}-x G\left(\sigma_{\mathrm{Z}}\right)
$$

have the same CSS $\sigma_{\mathrm{Z}}$. This is an important example in our analysis as all low-rank states discussed in Sec. VI are special cases of (30). Note that it is required to assume $x \leq x_{\max }^{\prime}=\left(R_{1}+R_{4}\right) / R_{1}$ to ensure that $\left(R_{4}-x \bar{R}_{4}\right) \geq 0$. Assuming for simplicity that $R_{1} \geq R_{4}$, the analogous conditions for $\left(R_{i}-x \bar{R}_{i}\right) \geq 0$ with $i=1,2,3$ are also satisfied for $x \leq x_{\max }^{\prime}$. On the other hand, the condition $\left(R_{2}-x \bar{R}_{2}\right)\left(R_{3}-x \bar{R}_{3}\right) \geq(Y-x \bar{Y})^{2}$, which is also implied by the positivity of $\rho_{\mathrm{Z}}$, restricts $x$ to be smaller than $x_{\max }^{\prime \prime}=f-\sqrt{f^{2}-4 \Delta \bar{\Delta}} /(2 \bar{\Delta})$, where $\Delta=R_{2} R_{3}-Y^{2}$, $\bar{\Delta}=\bar{R}_{2} \bar{R}_{3}-\bar{Y}^{2}$, and $f=R_{2} \bar{R}_{3}+\bar{R}_{2} R_{3}-2 Y \bar{Y}$. Thus, (301) is defined for $0<x \leq x_{\max } \equiv \min \left\{x_{\max }^{\prime}, x_{\max }^{\prime \prime}\right\}$. The problem of expressing $\sigma_{\mathrm{Z}}$ in terms of $\rho_{\mathrm{Z}}$ will be addressed in the following section.

\section{FORMULA APPLICATIONS FOR LOWER-RANK CSS}

Our compact-form formula can also be applied for lower-rank CSSs $\sigma$ in two approaches: directly for some special states and indirectly for arbitrary states.

To justify a direct application, the following conditions should be satisfied: (i) There must exist a full-rank edge state $\sigma^{\prime}$ in the vicinity of $\sigma$. If it is certain that $\sigma$ is a CSS for some $\rho$, this condition is trivial. However, if we do not know whether or not $\sigma$ can be a CSS for some $\rho$, this is not trivial. (ii) Let $\left|\phi^{\prime}\right\rangle$ be the kernel of $\sigma^{\prime \Gamma}$, i.e., $\sigma^{\prime \Gamma}\left|\phi^{\prime}\right\rangle=$ 0 . Then, $\left|\phi^{\prime}\right\rangle$ must be entangled. However, when $\left|\phi^{\prime}\right\rangle$ is not entangled, we merely cannot find any entangled $\rho^{\prime} \geq 0$ for $\sigma^{\prime}$ by the compact-form formula, and hence this condition is not so important. (iii) There must exist a sequence such that $\sigma^{\prime} \rightarrow \sigma$ and $\left|\phi^{\prime}\right\rangle \rightarrow|\phi\rangle$ simultaneously. This condition seems to severely constrain the choice of $|\phi\rangle$ in the case of $\operatorname{rank}\left(\sigma^{\Gamma}\right)=2$.

Thus, our formula can be applied directly to the rank-2 Horodecki state defined for $p \in\langle 0,1\rangle$ by [22]

$$
\rho_{\mathrm{H}}=p\left|\psi^{( \pm)}\right\rangle\left\langle\psi^{( \pm)}|+(1-p)| 00\right\rangle\langle 00|,
$$

which is a mixture of a Bell state $\left|\psi^{( \pm)}\right\rangle=(|01\rangle \pm$ $|10\rangle) / \sqrt{2}$ and a separable state orthogonal to it. It is worth noting that the Horodecki state is extremal in the sense that it minimizes the REE for a given concurrence [9], negativity (i.e., a measure of the PPT entanglement cost) for a given concurrence [9], fidelity (i.e., maximal singlet fraction) for a given concurrence $(\geq 1 / 3)$, and negativity $[\geq(\sqrt{5}-2) / 3][25]$. The state also satisfies some extremal conditions for the REE for a given negativity [15]. The CSS for $\rho_{\mathrm{H}}$ derived from (3) - (5) reads as

$$
\sigma_{\mathrm{H}}=q^{\prime 2}|00\rangle\left\langle 00\left|+2 p^{\prime} q^{\prime}\right| \psi^{( \pm)}\right\rangle\left\langle\psi^{( \pm)}\left|+p^{\prime 2}\right| 11\right\rangle\langle 11|,
$$

where $p^{\prime}=p / 2$ and $q^{\prime}=1-p^{\prime}$, in agreement with the known result derived in another way [2]. Note that, although $\operatorname{rank}\left(\sigma_{H}^{\Gamma}\right)=3, \sigma_{\mathrm{H}}$ is not full rank. Fortunately, the above-mentioned conditions necessary for the direct application of the compact-form formula are satisfied.

In the second more general approach, one can apply our formula for arbitrary lower-rank states in a limiting sequence from a full-rank state because the REE is a continuous function. For example of such application of our formula for lower-rank states we will analyze pure states and the rank-2 Vedral-Plenio state defined by [2]:

$$
\rho_{\mathrm{VP}}=p\left|\psi^{(+)}\right\rangle\left\langle\psi^{(+)}|+(1-p)| 01\right\rangle\langle 01|
$$

for $0 \leq p \leq 1$ with the corresponding CSS

$$
\sigma_{\mathrm{VP}}=\left(1-\frac{p}{2}\right)|01\rangle\left\langle 01\left|+\frac{p}{2}\right| 10\right\rangle\langle 10|,
$$

for which $\operatorname{rank}\left(\sigma_{\mathrm{VP}}\right)=\operatorname{rank}\left(\sigma_{\mathrm{VP}}^{\Gamma}\right)=2$. By contrast with the Horodecki state, (33) is a mixture of a Bell state and a separable state not orthogonal to it.

To derive CSSs $\sigma_{\mathrm{P}}$ and $\sigma_{\mathrm{VP}}$, and thus to show the usefulness of our formula also for lower-rank states, let us apply state $\sigma_{\mathrm{Z}}$, given by (24), in the limiting cases. Namely, by assuming in (30) that $x=x_{\max }^{\prime} \leq x_{\max }^{\prime \prime}$ and $R_{1} \geq R_{4}$, one gets the following extremal state:

$$
\rho_{\mathrm{Z}}^{\prime} \equiv \rho_{\mathrm{Z}}\left(x=x_{\max }^{\prime}\right)=\left(\begin{array}{cccc}
r_{1} & 0 & 0 & 0 \\
0 & r_{2} & y & 0 \\
0 & y & r_{3} & 0 \\
0 & 0 & 0 & 0
\end{array}\right),
$$


where

$$
\begin{aligned}
r_{1} & =R_{1}-R_{4}, \\
r_{2} & =R_{2}+\frac{2 R_{4}}{z^{2}}\left(R_{2}^{2}-R_{2} R_{3}+2 Y^{2}\right)+\frac{2 R_{4}}{L z}\left(R_{2}-R_{3}\right), \\
r_{3} & =1-r_{1}-r_{2}, \\
y & =\frac{1}{2 Y}\left[2\left(R_{1}+R_{2}\right) R_{4}-\left(r_{2}-R_{2}\right)\left(R_{2}-R_{3}\right)\right] .
\end{aligned}
$$

In the special case of $\sigma_{\mathrm{Z}}^{\prime}$ for $R_{1}=R_{4} \rightarrow 0$, one gets

$$
\sigma_{\mathrm{Z}}^{\prime \prime}=\left(1-R_{3}\right)|01\rangle\left\langle 01\left|+R_{3}\right| 10\right\rangle\langle 10|,
$$

then $\rho_{\mathrm{Z}}^{\prime \prime}=\sigma_{\mathrm{Z}}^{\prime \prime}-x \bar{Y}(|01\rangle\langle 10|+| 10\rangle\langle 01|)$ with $\bar{Y}=-(1-$ $\left.2 R_{3}\right) /\left[4 \operatorname{atanh}\left(1-2 R_{3}\right)\right]$, where atanh is the inverse hyperbolic tangent. This state for $x=R_{3} / \bar{Y}$ assuming $R_{3} \leq 1 / 2$ corresponds to a generalized Vedral-Plenio state:

$$
\rho_{\mathrm{GVP}}=p\left|\psi_{P}\right\rangle\left\langle\psi_{P}|+(1-p)| 01\right\rangle\langle 01|,
$$

where in comparison with the standard Vedral-Plenio state $\rho_{\mathrm{VP}}$, given by (33), a Bell state is replaced by a pure state

$$
\left|\psi_{P}\right\rangle=\sqrt{P}|01\rangle+e^{i \varphi} \sqrt{1-P}|10\rangle,
$$

for any $0 \leq P \leq 1$. For simplicity we set $\varphi=0$. Thus, the CSS $\sigma_{\mathrm{GVP}}$ is just given by Eq. (37) for $R_{3}=p(1-P)$. By assuming $R_{3}=p / 2$ and $x=R_{3} / \bar{R}$, one gets $\rho_{\mathrm{VP}}$ and its CSS $\sigma_{\mathrm{VP}}$, given by (34), in agreement with the solution obtained in Ref. [2]. Moreover, any two-qubit pure state $|\psi\rangle=c_{0}|00\rangle+c_{1}|01\rangle+c_{2}|10\rangle+c_{3}|11\rangle$ can be transformed by local rotations into the state (39). Thus, the CSS $\sigma_{\mathrm{Z}}^{\prime \prime}$, given by (37), also describes the CSS $\sigma_{\mathrm{P}}$ for an arbitrary pure state $|\psi\rangle$ as expected 1].

A mixed state introduced by Gisin [23],

$$
\rho_{\mathrm{G}}=q|00\rangle\left\langle 00|+p| \psi_{P}\right\rangle\left\langle\psi_{P}|+q| 11\right\rangle\langle 11|,
$$

where $\left|\psi_{P}\right\rangle$ is given by (39), $0 \leq p \leq 1$, and $q=(1-p) / 2$. By contrast to the generalized Vedral-Plenio state and the generalized Horodecki state, (40) is a mixture of an entangled pure state and two separable states orthogonal to it. The Gisin state is also a special case of $\rho_{\mathrm{Z}}$ assuming $R_{1}-x \bar{R}_{1}=R_{4}-x \bar{R}_{4}=q$. Thus, its CSS is equal to $\sigma_{\mathrm{Z}}$, given by (24) for $R_{1}=R_{4}$. It is worth noting that (40) is one of the simplest examples of an entangled state, which does not violate any Bell-type inequality (for some range of $p$ for a given $P$ ) 24].

Assuming $y^{2}=r_{2} r_{3}$, the state $\rho_{\mathrm{Z}}^{\prime}$ reduces to a rank2 state, which we refer to as the generalized Horodecki state defined as

$$
\rho_{\mathrm{GH}}=p\left|\psi_{P}\right\rangle\left\langle\psi_{P}|+(1-p)| 00\right\rangle\langle 00|,
$$

where $p=1-r_{1}$ and $\left|\psi_{P}\right\rangle$ is given by (39) for $P=$ $r_{2} /\left(1-r_{1}\right)$. Note that, for $P=1 / 2$, which corresponds to $r_{2}=r_{3}, \rho_{\mathrm{GH}}$ reduces to the standard Horodecki state $\rho_{\mathrm{H}}$ given in terms of a Bell state $\left|\psi_{P}\right\rangle=\left|\psi^{(+)}\right\rangle$. On the other hand, the state $\rho_{\mathrm{GH}}$ for $r_{1}=(1+C) / 2$ and $r_{2}=$ $d_{+}$reduces to the Verstraete-Verschelde state defined for $C \leq 1 / 3$ by 25 ]

$$
\rho_{\mathrm{V}}=\left(\begin{array}{llll}
\frac{1+C}{2} & 0 & 0 & 0 \\
0 & d_{+} & \frac{C}{2} & 0 \\
0 & \frac{C}{2} & d_{-} & 0 \\
0 & 0 & 0 & 0
\end{array}\right)
$$

where $d_{ \pm}=\frac{1}{4}\left(1-C \pm \sqrt{1-2 C-3 C^{2}}\right)$ and $C \equiv C\left(\rho_{\mathrm{V}}\right)$ is the concurrence, which can be expressed in terms of negativity $N=N\left(\rho_{\mathrm{V}}\right)$, as $C=\frac{1}{2}[N+\sqrt{N(4+5 N)}]$ holds. It is worth noting that state minimizes fidelity for a given concurrence and negativity [25].

It is seen by analyzing $\rho_{Z}^{\prime}$ as a function of elements of $\sigma_{Z}^{\prime}$ that it seems impossible to invert the general Eqs. (35) and (36). However, the equations can be inverted in some special cases. For example, by assuming $R_{2}=R_{3}$ for the state $\rho_{Z}^{\prime}$, which implies $r_{2}=r_{3}=\left(1-r_{1}\right) / 2$, one finds that the general relation for $r_{2}$ in Eqs. (36) reduces to $r_{2}=R_{2}+R_{4}=1-R_{1}-R_{2}$. Under this assumption, the set of equations (36) can be solved for $\left\{R_{i}\right\}$ in terms of $\left\{r_{i}\right\}$ and $y \leq r_{2}$ as follows:

$$
\begin{aligned}
R_{4} & =\frac{4 r_{1} y^{2}}{\left(1+r_{1}\right)^{2}-4 y^{2}} \\
R_{2} & =R_{3}=r_{2}-R_{4} \\
R_{1} & =r_{1}+R_{4}
\end{aligned}
$$

This state, in the special case of $r_{2}=y$, reduces to the standard Horodecki state $\rho_{\mathrm{H}}$, given by (31). However, it does not reduce to the generalized Horodecki state $\rho_{\mathrm{GH}}$ if $P \neq \frac{1}{2}$, for which we can give only a formal solution for the REE:

$$
E_{R}^{(\mathrm{GH})}=-H_{2}\left(r_{1}\right)-r_{1} \log R_{1}-f_{-}^{2} \log \lambda_{-}-f_{+}^{2} \log \lambda_{+},
$$

where $H_{2}(\cdot)$ is the binary entropy and $f_{ \pm}=\mathcal{N}_{ \pm}\left[\left(\lambda_{ \pm}-\right.\right.$ $\left.\left.R_{3}\right) \sqrt{r_{2}}+Y \sqrt{r_{3}}\right]$, while $\lambda_{ \pm}$and $\mathcal{N}_{ \pm}$are defined below Eq. (25). A compact-form explicit formula for the REE for the states $\sigma_{Z}^{\prime}$ with elements given by (43) as well as for the Horodecki state or the Vedral-Plenio state is thus obtained. However, it seems impossible to invert (36) in order to express all $\left\{R_{i}\right\}$ in terms of $\left\{r_{i}\right\}$ and $y$, even for the generalized Horodecki state with $P, p \neq 0, \frac{1}{2}, 1$.

\section{NONUNIQUENESS OF THE CSS FOR BELL STATES}

Analysis of our formula and the above examples enables us to find that for a given entangled state there is not always a unique CSS due to a limiting procedure. For this purpose let us derive CSSs for a Bell state $\left|\psi^{(+)}\right\rangle$ from $\sigma_{\mathrm{Z}}$, given by (24). First, assume that $R_{1}=R_{4}=\epsilon$ and $R_{2}=R_{3}=1 / 2-\epsilon$ for small $\epsilon \geq 0$. By taking the limit of $\epsilon \rightarrow 0$, one gets the following CSS:

$$
\sigma_{\text {Bell }}^{\prime}=\lim _{\epsilon \rightarrow 0} \sigma_{\mathrm{Z}}=\frac{1}{2}(|01\rangle\langle 01|+| 10\rangle\langle 10|),
$$


for which $G\left(\sigma_{\text {Bell }}^{\prime}\right)=-\frac{1}{4}(|01\rangle\langle 10|+| 10\rangle\langle 01|)$. By noting that $x_{\max }=2$, one finds that $\sigma_{\text {Bell }}^{\prime}-x_{\max } G\left(\sigma_{\text {Bell }}^{\prime}\right)$ corresponds to the Bell state $\left|\psi^{(+)}\right\rangle$. The same CSS is obtained from special cases of the CSS for pure state and the Vedral-Plenio state, given by (34) for $p=1$. On the other hand, let us analyze $\sigma_{\mathrm{Z}}$ assuming $R_{i}=1 / 4-\epsilon$ for $i=1, \ldots, 4$. Then, in the limit of $\epsilon \rightarrow 0, \sigma_{\mathrm{Z}}$ reduces to the CSS:

$\sigma_{\text {Bell }}^{\prime \prime}=\lim _{\epsilon \rightarrow 0} \sigma_{\mathrm{Z}}=\frac{1}{4}\left(|00\rangle\left\langle 00|+2| \psi^{(+)}\right\rangle\left\langle\psi^{(+)}|+| 11\right\rangle\langle 11|\right)$,

for which state $\sigma_{\text {Bell }}^{\prime \prime}-x_{\max } G\left(\sigma_{\text {Bell }}^{\prime \prime}\right)$ also corresponds to the Bell state $\left|\psi^{(+)}\right\rangle$. Note that (46) is a special case of the CSS for the Horodecki state, given by (32) for $p=1$, and for the Gisin state, given by (40) for $p=P=\frac{1}{2}$.

In a more general approach, let us analyze a Belldiagonal state of the form

$$
\rho_{\mathrm{BD}}=(1-k \epsilon)\left|\beta_{1}\right\rangle\left\langle\beta_{1}\left|+\epsilon \sum_{i=2}^{4} k_{i}\right| \beta_{i}\right\rangle\left\langle\beta_{i}\right|,
$$

where $0 \leq k_{i}<\infty$ and $k \equiv k_{2}+k_{3}+k_{4}$ such that $k \epsilon \leq 1$. The state in the limit of $\epsilon \rightarrow 0$ reduces to the Bell state $\left|\beta_{1}\right\rangle$, for which the CSS depends on $\left\{k_{i}\right\}$ as follows:

$$
\sigma_{\text {Bell }}=\frac{1}{2}\left|\beta_{1}\right\rangle\left\langle\beta_{1}\left|+\frac{1}{2 k} \sum_{i=2}^{4} k_{i}\right| \beta_{i}\right\rangle\left\langle\beta_{i}\right|
$$

according to (23). Thus, an arbitrary Bell-diagonal state with one of its eigenvalues equal to $1 / 2$ is the CSS for a Bell state. In special cases, $\sigma_{\text {Bell }}$ goes to (45) for $k_{2}=$ $1, k_{3}=k_{4}=0$ and to (46) for $k_{2}=0, k_{3}=k_{4}=1$ assuming $\left|\beta_{1,2}\right\rangle=\left|\psi^{( \pm)}\right\rangle$and $\left|\beta_{3,4}\right\rangle=\left|\phi^{( \pm)}\right\rangle=\frac{1}{\sqrt{2}}(|00\rangle \pm$ $|11\rangle)$. Other CSSs of Bell states, which are not diagonal in the Bell basis, can be obtained by rotating $\sigma_{\text {Bell }}$. For example, the CSS

$$
\sigma_{\text {Bell }}^{\prime \prime \prime}=\frac{1}{4}\left(2\left|\psi^{(+)}\right\rangle\left\langle\psi^{(+)}|+| \psi\right\rangle\left\langle\psi|+| \phi^{(-)}\right\rangle\left\langle\phi^{(-)}\right|\right),
$$

where $|\psi\rangle=\frac{1}{\sqrt{2}}\left(\left|\psi^{(-)}\right\rangle+\left|\phi^{(+)}\right\rangle\right)$, is obtained by rotating $\sigma_{\text {Bell }}^{\prime \prime}$ given by (46).

It is worth noting that the generated Bell-diagonal state $\rho=\sigma_{\text {Bell }}-x G\left(\sigma_{\text {Bell }}\right)$ is independent of the parameters $\left\{k_{i}\right\}$ in $\sigma_{\text {Bell }}$ only for $x=x_{\max }$, but it depends on the choice of $\left\{k_{i}\right\}$ for $x<x_{\max }$, although the largest eigenvalue of $\rho, \lambda_{1}=\max (\operatorname{eig} \rho)$, is $\left\{k_{i}\right\}$ independent. Thus, the REE is also independent of $\left\{k_{i}\right\}$, as
$E_{\mathrm{R}}(\rho)=1-H_{2}\left(\lambda_{1}\right)$ for $\lambda_{1} \geq \frac{1}{2}$ and $E_{\mathrm{R}}(\rho)=0$ otherwise. Similarly, other entanglement measures, including the negativity and concurrence are $\left\{k_{i}\right\}$ independent, as $N(\rho)=C(\rho)=2 \lambda_{1}-1$. By contrast, violation of Bellinequality by $\rho$ depends on all eigenvalues $\left\{\lambda_{i}\right\}$, so it depends on the choice of $\left\{k_{i}\right\}$ for $\sigma_{\text {Bell }}$. This can be seen explicitly, by analyzing the Horodecki parameter $M$ 27, 28] describing a degree of the violation of the Bell inequality due to Clauser, Horne, Shimony, and Holt.

\section{CONCLUSION}

We have addressed the long-standing problem of finding a qubit formula for the relative entropy of entanglement [18] or, equivalently, of finding the CSS $\sigma$ for a given entangled state $\rho$. We have obtained a solution to the inverse problem by finding a compact expression for an entangled state $\rho$ for a given $\operatorname{CSS} \sigma$, which is a crucial simplification of the former solution [13]. The usefulness of our formula can be demonstrated by finding the REE for some special states but also by analyzing general properties of the REE. Thus, we have studied (i) weak and strong additivity of the REE, (ii) how the REE is related to the Rains upper bound for the entanglement of distillation, and (iii) nonuniqueness of the closest separable states for Bell states.

All the examples of entangled states $\rho$ with analytical expression for the CSSs $\sigma$, discussed in Refs. 1, 2, 8, 9, 10, 11, 15, 16], can easily be explained by using our formula as follows. By starting from some special $\sigma$ one should generate $\rho$ and then try to find an inverse analytical relation to express $\sigma$ in terms of $\rho$. Thus, for example, we have derived the well-known formulas for pure, Bell-diagonal states, the Horodecki states 22], the Vedral-Plenio states [2], and the Gisin states [23] but also obtained new formulas for some other entangled states.

We have analyzed more general states $\rho_{\mathrm{Z}}$ with elements $\left\{r_{i}\right\}$, which can be generated from the CSS $\sigma_{\mathrm{Z}}$ with elements $\left\{R_{i}\right\}$ via Eqs. (36). The point is that, apart from some special cases including $R_{2}=R_{3}$ and $R_{1}=R_{4}=0$, the set of Eqs. (36) for $\left\{r_{i}\right\}$ as a function of $\left\{R_{i}\right\}$ seemingly cannot be solved for $\left\{R_{i}\right\}$ due to the presence of logarithmic functions (29). Thus, we cannot express $\sigma_{Z}$ in terms of $\rho_{Z}$ in general. Although this is not a proof of impossibility, our analysis of the formula for $\rho_{\mathrm{Z}}$ in terms of $\sigma_{\mathrm{Z}}$ strongly suggests that the inverse problem, which corresponds to finding a compact-form relation for $\sigma$ in terms of a given $\rho$, can be solved in some special cases only.
[1] V. Vedral, M. B. Plenio, M. A. Rippin, and P. L. Knight, Phys. Rev. Lett. 78, 2275 (1997).

[2] V. Vedral and M. B. Plenio, Phys. Rev. A 57, 1619 (1998).

[3] M. J. Donald and M. Horodecki, Phys. Lett. A 264, 257 (1999).
[4] M. B. Plenio, S. Virmani and P. Papadopoulos, J. Phys. A 33, L193 (2000).

[5] K. M. R. Audenaert, J. Eisert, E. Jané, M. B. Plenio, S. Virmani, and B. De Moor, Phys. Rev. Lett. 87, 217902 (2001).

[6] E. M. Rains, IEEE Trans. Inf. Theory 47, 2921 (2001). 
[7] K. G. H. Vollbrecht and R. F. Werner, Phys. Rev. A 64, 062307 (2001).

[8] F. Verstraete, K. Audenaert, and B. De Moor, Phys. Rev. A 64, 012316 (2001).

[9] F. Verstraete, K. M. R. Audenaert, J. Dehaene, and B. De Moor, J. Phys. A 34, 10327 (2001).

[10] F. Verstraete, J. Dehaene, and B. De Moor, J. Mod. Opt. 49, 1277 (2002).

[11] K. M. R. Audenaert, B. De Moor, K. G. H. Vollbrecht, and R. F. Werner, Phys. Rev. A 66, 032310 (2002).

[12] S. Ishizaka, J. Phys. A 35, 8075 (2002).

[13] S. Ishizaka, Phys. Rev. A 67, 060301(R) (2003).

[14] S. Ishizaka, Phys. Rev. A 69, 020301(R) (2004).

[15] A. Miranowicz and A. Grudka, J. Opt. B: Quantum Semiclassical Opt. 6, 542 (2004).

[16] T. C. Wei, M. Ericsson, P. Goldbart,and W. J. Munro, Quantum Inf. Comput. 4, 252 (2004).

[17] R. Horodecki, P. Horodecki, M. Horodecki and K. Horodecki, e-print arXiv:quant-ph/0702225

[18] J. Eisert, Problem 8: Qubit formula for relative entropy of entanglement, in: Some Open Problems in Quantum Information Theory, eds. O. Krueger, R.F. Werner, e- print quant-ph/0504166 v1.

[19] J. Řehaček and Z. Hradil, Phys. Rev. Lett. 90, 127904 (2003).

[20] M. Lewenstein, B. Kraus, J. I. Cirac, and P. Horodecki, Phys. Rev. A 62, 052310 (2000).

[21] F. Verstraete, J. Dehaene, and B. De Moor, Phys. Rev. A 64, 010101(R) (2001).

[22] M. Horodecki, P. Horodecki, and R. Horodecki, in Quantum Information: An Introduction to Basic Theoretical Concepts and Experiments, edited by G. Alber et al. (Springer, Berlin, 2001), p. 151.

[23] N. Gisin, Phys. Lett. A 210, 151 (1996).

[24] A. Peres, Phys. Rev. Lett. 77, 1413 (1996).

[25] F. Verstraete and H. Verschelde, Phys. Rev. A 66, 022307 (2002).

[26] E. M. Rains, Phys. Rev. A 60, 179 (1999); 63, 019902(E) (2000).

[27] R. Horodecki, P. Horodecki, and M. Horodecki, Phys. Lett. A 200, 340 (1995).

[28] A. Miranowicz, Phys. Lett. A 327, 272 (2004). 